\title{
ARTICLE
}

Received 23 Jul 2013 | Accepted 29 Oct 2013 | Published 25 Nov $2013 \quad$ DOl: 10.1038/ncomms3844

\section{General nature of liquid-liquid transition in aqueous organic solutions}

\author{
Ken-ichiro Murata ${ }^{1} \&$ Hajime Tanaka ${ }^{1}$
}

The presence or absence of a liquid-liquid transition in water is one of the hot topics in liquid science, and while a liquid-liquid transition in water/glycerol mixtures is known, its generality in aqueous solutions has remained elusive. Here we reveal that 14 aqueous solutions of sugar and polyol molecules, which have an ability to form hydrogen bonding with water molecules, exhibit liquid-liquid transitions. We find evidence that both melting of ice and liquid-liquid transitions in all these aqueous solutions are controlled solely by water activity, which is related to the difference in the chemical potential between an aqueous solution and pure water at the same temperature and pressure. Our theory shows that water activity is determined by the degree of local tetrahedral ordering, indicating that both phenomena are driven by structural ordering towards ice-like local structures. This has a significant implication on our understanding of the low-temperature behaviour of water.

\footnotetext{
${ }^{1}$ Institute of Industrial Science, University of Tokyo, 4-6-1 Komaba, Meguro-ku, Tokyo 153-8505, Japan. Correspondence and requests for materials should be addressed to H.T. (email: tanaka@iis.u-tokyo.ac.jp).
} 
W ater exhibits many anomalous behaviours upon cooling, which include volume expansion $\left(<4{ }^{\circ} \mathrm{C}\right)$, softening $\left(<46^{\circ} \mathrm{C}\right)$ and heat capacity increase $\left(<35^{\circ} \mathrm{C}\right)^{1-4}$. These features are absent in other molecular liquids. These are the unique properties that make water very special and important in nature ${ }^{1-4}$. The unusual features of liquid water are more enhanced at lower temperatures and lower pressures, reflecting enhanced tetrahedral structures stabilized by hydrogen bonding. There is a consensus that the unique properties of water come from local tetrahedral ordering due to hydrogen bonding. This is supported by the fact that atomic liquids having similar tetrahedral ordering due to covalent bonding exhibit water-like anomalies and phase behaviours ${ }^{5}$. However, the very origin of the water anomalies is still a matter of debate and far from complete understanding ${ }^{1,6-9}$.

One of the most striking phenomena expected to exist in water is a liquid-liquid transition (LLT): a first-order transformation from one liquid to another even in a single-component liquid. LLT between low-density and high-density liquid water was suggested from the experimental finding of the two distinct amorphous forms of water and the first-order-like transition between them ${ }^{10}$. The low-density amorphous ice is called LDA, whereas the high-density one HDA. The solid-state transition between LDA and HDA has a mechanical nature, which obscures its connection to the thermodynamic transition between two liquids ${ }^{11}$. Triggered by this finding, computer simulation studies have indeed indicated its presence in a highly supercooled state of water ${ }^{2,3,12}$, although there is an ongoing debate on the interpretation ${ }^{13-18}$. Unfortunately, however, there is no experimental access to LLT in bulk liquid water, because LLT is considered to exist in the so-called 'no man's land', that is, a region below a homogeneous nucleation temperature $T_{\mathrm{H}}(235 \mathrm{~K}$ at ambient pressure) and above the freezing point of ultraviscous water to cubic ice, where water exists only in its crystalline states ${ }^{3}$. So exploring the low-temperature region of liquid water is of fundamental importance.

Although it is almost impossible to access 'no man's land' for bulk pure water, there are still some indirect ways to access the low-temperature behaviour of liquid water. One is to use nanoconfined water. The confinement of water in a very small hydrophilic cavity (typically, nanometre-scale) indeed suppresses crystallization of water to ice and allows accessing the no man's land (for example, refer to the study by Mallamace et al. ${ }^{19}$ ). However, surface chemistry and confinement effects have key roles in the behaviour of confined water and might affect the state of water in a fundamental manner ${ }^{20,21}$. The other method we recently employed ${ }^{22}$ was to mix water with solutes that prevent ice crystallization and lower the upper bound of no man's land ${ }^{23,24}$. We chose organic solutes that have a capability to form hydrogen bonding with water molecules and efficiently lower the homogeneous nucleation line. By mixing water with glycerol, which is a famous anti-freezing agent, we found LLT in the mixture ${ }^{22}$. Although it is suggestive of the existence of LLT in pure water, there is of course a possibility that this LLT occurs only in the presence of glycerol and not in pure water. To clarify this point, it is essential to study how general this LLT is to various aqueous solutions.

We note that LLT observed in water/glycerol mixtures did not accompany demixing. Namely, it is a genuine transition from homogeneous liquid I to liquid II, that is, a transition between two liquids with identical compositions but different densities. In principle, however, there is a possibility of phase demixing associated with LLT for binary mixtures ${ }^{25}$. The relationship between demixing transitions between two liquid phases of different composition in aqueous systems, and a possible underlying LLT between phases of identical composition was first pointed out by Angell and Sare ${ }^{26}$ and also theoretically studied by Chatterjee and Debenedetti ${ }^{27}$. Recently such LLTinduced demixing was suggested by Mishima and Suzuki ${ }^{28}$ for aqueous solutions of $\mathrm{LiCl}$ and shown by numerical simulation ${ }^{29}$. So it is natural to ask what physical factor determines whether LLT accompanies demixing or not.

When water is mixed with other miscible components, the unique characteristics of water are generally weakened. How aqueous solutions still keep water-like characters is expressed by the water activity, $a_{\mathrm{w}}$. This quantity is related to the difference in the chemical potential between an aqueous solution and pure water at the same temperature and pressure ${ }^{23}$ : the smaller the difference, the higher water activity. Experimentally, it is characterized by the amount of the melting point depression as a function of a solute concentration, $c$. Ice crystals formed in water-rich aqueous solutions are made of $100 \%$ water molecules in usual conditions and, accordingly, the free energy of the ice is not perturbed by the solute molecules in a mixture. Thus the melting point $T_{\mathrm{m}}$ of the ice crystal is primarily determined by the free energy of the liquid state, which is affected by the presence of solute molecules that perturbs hydrogen-bond network. In the dilute limit, the water activity is proportional to the fraction of water molecules, $1-c$. This suggests that by revealing the relation between the water activity and LLT it may be possible to obtain crucial information on the question of whether LLT exists in pure water (that is, in the limit of $c \rightarrow 0$ ) or not.

Thus, to address the generality of LLT in aqueous solutions, a physical factor controlling whether LLT accompanies phase demixing or not, and the relation between the water activity and LLT, we perform a systematic study of the low-temperature behaviours of aqueous solutions of various sugars and polyols, which have cryoprotective capability, and find that 14 aqueous solutions of sugar and polyol molecules exhibit LLTs. We show that both melting of ice and LLT in all these aqueous solutions are controlled solely by water activity and provide a theoretical explanation for this finding. We expect that the understanding of the phase behaviour of such aqueous solutions may bring new insight into the very nature of water, which is important in a wide range of fields related to water, including (cryo)biological ${ }^{30}$, climate $^{31}$, geological and astrophysical science ${ }^{32}$.

\section{Results}

Examples of LLT without demixing. First, we describe the phase transition dynamics of LLT without demixing. Figure 1a,b shows, respectively, nucleation and growth (NG)- and spinodal decomposition (SD)-type transformation during LLT without demixing, which were observed in 1,2,4-butanetriol/water solution (the molar fraction of the solute, $c=0.155$ ) with phase-contrast microscopy (see Methods). For NG-type LLT observed at the annealing temperature $T_{\mathrm{a}}=184.5 \mathrm{~K}$, the liquid II phase is nucleated as droplets in the matrix of liquid I and their size grows linearly with time. For SD-type LLT observed at $T_{\mathrm{a}}=178 \mathrm{~K}$, on the other hand, liquid I transforms into liquid II continuously, accompanying spontaneous growth of spatial fluctuations of the order parameter (see spatial fluctuations in the image at $20 \mathrm{~min}$ in Fig. 1b). These behaviours are basically the same as those observed in LLT of triphenyl phosphite (TPP) ${ }^{33}, n$-butanol ${ }^{34}$ and water/glycerol mixtures ${ }^{22}$. This suggests the universality of the pattern evolution in LLT for molecular liquids.

The kinetic pathway of the transition is consistent with the cooperative ordering of a non-conserved order parameter, which may be the fraction of locally favoured tetrahedral-like structures of water, $S$, according to our two-order parameter model ${ }^{35,36}$. In our model, LLT is the first-order transition between a liquid state with low $S$ and that with high $S$. Thus, the initial liquid state 
a

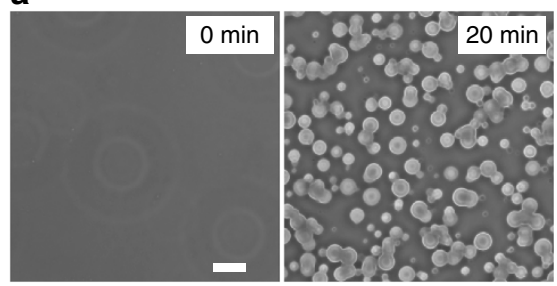

b
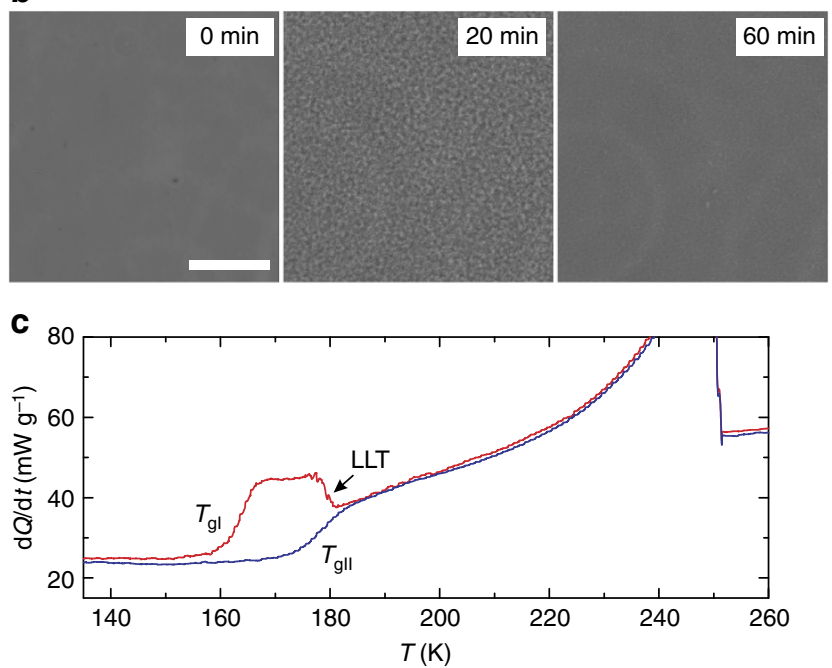

d

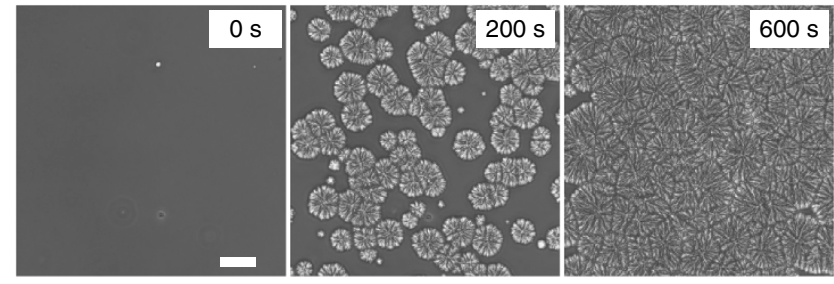

e

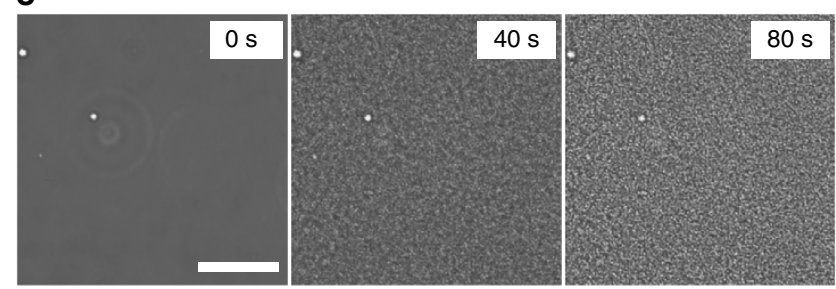

f

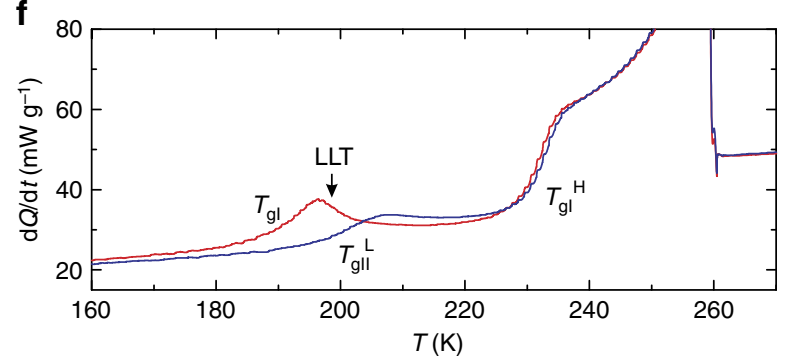

Figure 1 | Pattern evolution in LLT and glass transition behaviour. (a) NG-type pattern evolution during LLT without demixing at $T=184.5 \mathrm{~K}$, observed in a water/1,2,4-butanetriol solution ( $c=0.155$ ) with phase contrast microscopy. The scale bar corresponds to $50 \mu \mathrm{m}$. (b) SD-type pattern evolution at $T=178 \mathrm{~K}$ for $c=0.155$. In both cases, the initial state is liquid I and the final state is liquid II. The scale bar corresponds to $50 \mu \mathrm{m}$. (c) The reversible heat flow of liquid I (red line) and liquid II formed at $T_{a}=173 \mathrm{~K}$ without demixing (blue line), measured by ac DSC measurements in the heating process (heating rate: $1 \mathrm{~K} \mathrm{~min}^{-1}$, frequency: $60 \mathrm{~s}$, amplitude: $0.16 \mathrm{~K}$ ). Stepwise changes around $160 \mathrm{~K}$ (liquid I, $T_{\mathrm{gl}}$ ) and $173 \mathrm{~K}$ (liquid II, $T_{\mathrm{gII}}$ ) are the onsets of the glass transition. The temperature width of the glass transition is $6 \mathrm{~K}$ and $10 \mathrm{~K}$ respectively for liquid I and II, indicating that liquid II is stronger (or less fragile) than liquid I. (d) NG-type pattern evolution during LLT with demixing at $T=211 \mathrm{~K}$, observed in a water/sucrose solution ( $c=0.070$ ). The scale bar corresponds to $50 \mu \mathrm{m}$. (e) SD-type pattern evolution at $T=203 \mathrm{~K}$ for $c=0.070$. In this case, the initial state is liquid I but the final state is demixed to liquid II with less solute and liquid I with more solute. The scale bar corresponds to $50 \mu \mathrm{m}$. (f) The reversible heat flow of liquid I (red line) and the final liquid II state with demixing formed at $T_{\mathrm{a}}=199 \mathrm{~K}$ (blue line), measured by ac DSC measurements in the heating process ( $c=0.081$ ). A stepwise change $\sim 188 \mathrm{~K}$ (red line) is the glass transition of liquid I ( $\left.T_{\mathrm{gl}}\right)$, whereas those $\sim 195 \mathrm{~K}$ and $230 \mathrm{~K}$ (blue line) are the glass transition of liquid II with less sucrose $\left(T_{\mathrm{g} \|}^{\mathrm{L}}\right)$ and liquid I with more sucrose $\left(T_{\mathrm{gl}}^{\mathrm{H}}\right)$, respectively.

(liquid I) is a liquid containing a low fraction of locally favoured structures (low-S liquid), whereas the final state is a liquid containing a high fraction of locally favoured structures (high-S liquid). For NG-type LLT, nucleation of droplets of the high-S liquid (liquid II) takes place by overcoming a barrier and then droplets grow until they fill up the space. For SD-type LLT, on the other hand, liquid I continuously transforms to liquid II without any barrier, accompanying the spontaneous growth of fluctuations of $S$ in the initial stage and the steady increase in the average value of $S$ until its saturation. The final state is macroscopically homogeneous for both cases, indicating the absence of demixing.

Here we mention other explanations proposed for the phenomena that we interpreted as LLT. Feldman et al. ${ }^{37}$ interpreted what we call liquid II as a mixture of the solute-rich liquid I phase, ices and interfacial water around ices, on the basis of dielectric spectroscopy measurements. Recently, Limmer and Chandler ${ }^{38}$ also suggested the possibility that the phenomena we interpret as LLT in a water/glycerol mixture may be a process of the liquid-to-crystal transition coupled with solute concentration fluctuations. This scenario predicts that for NG-type evolution droplets are rich in ice and interfacial water, whereas the matrix is rich in glycerol. However, these scenarios are difficult to explain the experimental facts that (i) there is only one glass transition for liquid II and (ii) the glycerol concentration is macroscopically homogeneous for both NG and SD-type LLT without demixing (see below and Supplementary Note 1). Thus, we argue that the transition we observe should be LLT, although further careful study may be necessary to settle this debate.

The homogeneous nature of the liquid II has also been confirmed by the calorimetric behaviour measured by ac DSC measurements (see Methods). Before showing the results, we explain the temperature protocols used in our DSC measurements for investigating calorimetric properties of liquid I and II. For liquid I (see Fig. 2a), an aqueous sample was quenched down to $123 \mathrm{~K}$ (below the glass transition temperature of liquid I) with the cooling rate of $20 \mathrm{~K} \mathrm{~min}^{-1}$ to avoid LLT during the cooling process and kept at this temperature for $15 \mathrm{~min}$. Then, ac DSC measurements were performed with the heating rate of $1 \mathrm{~K}$ $\mathrm{min}^{-1}$ (frequency: $60 \mathrm{~s}$, amplitude: $0.16 \mathrm{~K}$ ). For a state formed after LLT (see Fig. 2b), a sample was quenched down to $T_{\mathrm{a}}$ at which LLT takes place, with the cooling rate of $20 \mathrm{~K} \mathrm{~min}^{-1}$ and annealed at $T_{\mathrm{a}}$ to complete LLT. After the completion of LLT, the sample was quenched again down to $123 \mathrm{~K}$ with a cooling rate of $5 \mathrm{~K} \mathrm{~min}^{-1}$. Then, after waiting for $15 \mathrm{~min}$, we performed ac DSC measurements with the same condition as in the case of liquid I. 

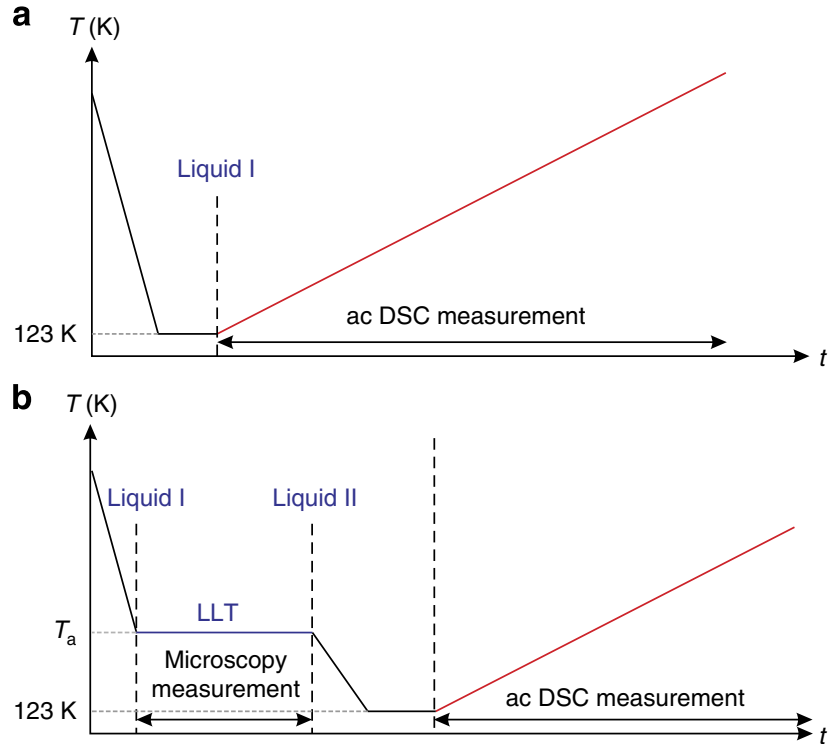

Figure 2 | Temperature protocols for microscopy and DSC

measurements. (a) The protocol used for liquid I. (b) The protocol used for a state formed after LLT, which is liquid II or liquid II coexisting with liquid I due to demixing. Microscopic observation was performed by annealing at a constant temperature $T_{\mathrm{a}}$ after a rapid temperature quench.

Figure 1c shows the reversible heat flow of liquid I and II obtained with ac measurements in the heating process (shown by the red lines in Fig. 2). We found only one glass transition in liquid II, $T_{\text {gII }}$, which is distinct from that of liquid I, $T_{\mathrm{gI}}$. Throughout this article, $T_{\mathrm{g}}$ means the lower edge of the heat signal due to the glass transition upon heating (that is, the onset of mobility). The presence of only one glass transition for liquid II strongly indicates that this transition should be LLT without demixing. Note that if the final state is composed of two phases, there should be two glass transitions. The temperature width of the glass transition is $6 \mathrm{~K}$ and $10 \mathrm{~K}$, respectively, for liquid I and II, meaning that liquid II is stronger (or less fragile) than liquid I (ref. 4).

Examples of LLT with demixing. Next, we show examples of the transformation process of LLT accompanying demixing. Figure 1d,e shows respectively NG- and SD-type transformation observed in a water/sucrose mixture $(c=0.070)$. The behaviour of the solutions of high molecular weight solutes is basically similar to this case. We also show the behaviour of LLT-induced demixing for aqueous solutions of low molecular weight solutes in Supplementary Fig. S1 and Supplementary Note 2. Although the kinetic behaviour resembles that of LLT without demixing, there are a few fundamental differences. First, we can see Mullins-Sekerka-type instability ${ }^{39}$ of the growth front of nuclei of liquid II (see Supplementary Fig. S2 and Supplementary Note 3). This indicates that a component that is not miscible with liquid II is expelled from the liquid II nuclei, inducing the concentration gradient on the growth front. This is further supported by the fact that the final state remains heterogeneous and never becomes homogeneous. In Fig. 1f, we show the reversible heat flow of liquid I and the final state formed after LLT $(c=0.081)$ in the heating process (see Fig. 2 for the temperature protocol and Supplementary Figs S3-S6, and Supplementary Note 4 and 5 for the detailed interpretation of the behaviour). We found two $T_{\mathrm{g}}$ 's for the final state, one of which is the low $T_{\mathrm{g}}$ of liquid II with less solute, $T_{\mathrm{gII}}^{\mathrm{L}}$, and the other is the high $T_{\mathrm{g}}$ of liquid I with more solute, $T_{\mathrm{gI}}^{\mathrm{H}}$ (see Fig. 5, Supplementary Fig. S7 and Supplementary Note 6 for the relevance of this assignment). The presence of the two $T_{\mathrm{g}}$ 's clearly indicates that this LLT accompanies demixing into two amorphous phases. The phenomena of LLT-induced demixing have also been confirmed by small- and wide-angle $\mathrm{X}$-ray scattering measurements (see below), which also indicate that liquid II contains nanometre-scale cubic ices. As ice $I_{c}$ itself has no glass transition despite its disordered nature ${ }^{40}$, we can exclude the possibility that the final state is merely a solute-rich liquid I coexisting with cubic ices $I_{c}$, as will be discussed below.

Here it is worth mentioning that the kinetics of the transition obeys the ordering of a non-conserved order parameter ${ }^{36}$ (as in magnetic ordering) even when accompanying demixing. The droplet growth mode (the domain size $l \sim t$ ) does not obey the coarsening law for the conserved order parameter $c$ (the domain size $l_{c} \sim t^{1 / 3}$ (ref. 41)), which is characteristic of simple demixing. This suggests that LLT, whose order parameter is non-conserved, has a primary role in the macroscopic domain formation during LLT. In other words, LLT proceeds first, then demixing follows. This indicates that liquid I is miscible with a solute, but liquid II is not. So LLT is necessary for demixing of these mixtures to take place. This is essentially the same as the case of a TPP/diethyl ether mixture ${ }^{25}$. We speculate that LLT-induced demixing in water/LiCl solutions ${ }^{28}$ also belongs to this category.

X-ray scattering evidence of LLT and demixing. The phenomena of LLT-induced demixing have also been confirmed by smalland wide-angle X-ray scattering (SWAXS) measurements. Here we show results of a water/xylitol solution $(c=0.125)$ as an example. In Fig. 3a, we plot the wide-angle X-ray scattering intensity $I(q)$ as a function of the wavenumber $q$ for homogeneous liquid I (less solute) and liquid II (more solute) coexisting with cubic ices. Here we can see a clear indication (a typical halo in $I(q)$ ) that liquid II contains primitive ice $\mathrm{I}_{c}$ unlike liquid I. By removing the contribution of ice $I_{c}$ from the total spectrum of liquid II (see Fig. 3b), we can reveal a hidden peak that is very similar to that of $\mathrm{LDA}^{42}$, as shown in Fig. 3c. This indicates that this transition is not just the formation of ice $I_{c}$ crystals but the transformation of liquid I to liquid II having LDA-like structures, even though it accompanies demixing.

In Fig. 3d, we show $I(q)$ of liquid II coexisting with liquid I in a small-angle regime. For $q>0.3 \mathrm{~nm}^{-1}$, we found the Guinier and Porod regime in $I(q)$, reflecting the presence of ice $\mathrm{I}_{c}$ crystals. The Porod law, $I(q) \propto q^{-4}$, tells us that ice $\mathrm{I}_{c}$ crystals have rather sharp interfaces. To analyse $I(q)$ in this regime, we employed a polydisperse sphere model with the Schultz-Zimm size distribution (see Methods). From this analysis, we estimated the characteristic radius of cubic ices, $r_{\mathrm{c}}$, as $2.5 \mathrm{~nm}$ and its variance $\sigma$ as 0.35 (see the inset of Fig. 3d). In addition to this signal from cubic ices, we also confirmed the presence of the excess scattering associated with another length scale in the range of $q<0.3 \mathrm{~nm}^{-1}$. We conclude that this scattering comes from phase-separated domains (see the schematic picture in Fig. 3d): the system consists of phase-separated domains, liquid II with less solute containing a small amount of ice $\mathrm{I}_{\mathrm{c}}$ and liquid I with more solute. The domain size is probably larger than $10 \mathrm{~nm}$ because the Guinier regime of the domain scattering is not observed in our $q$ range. We note that droplet nuclei observed in Fig. 1d have these small domains as their internal structures: the size of droplet nuclei $>>$ phase-separated domains. To access the detailed characteristics of the domain structure (for example, size and dimension), ultra small-angle X-ray scattering measurements are necessary. 

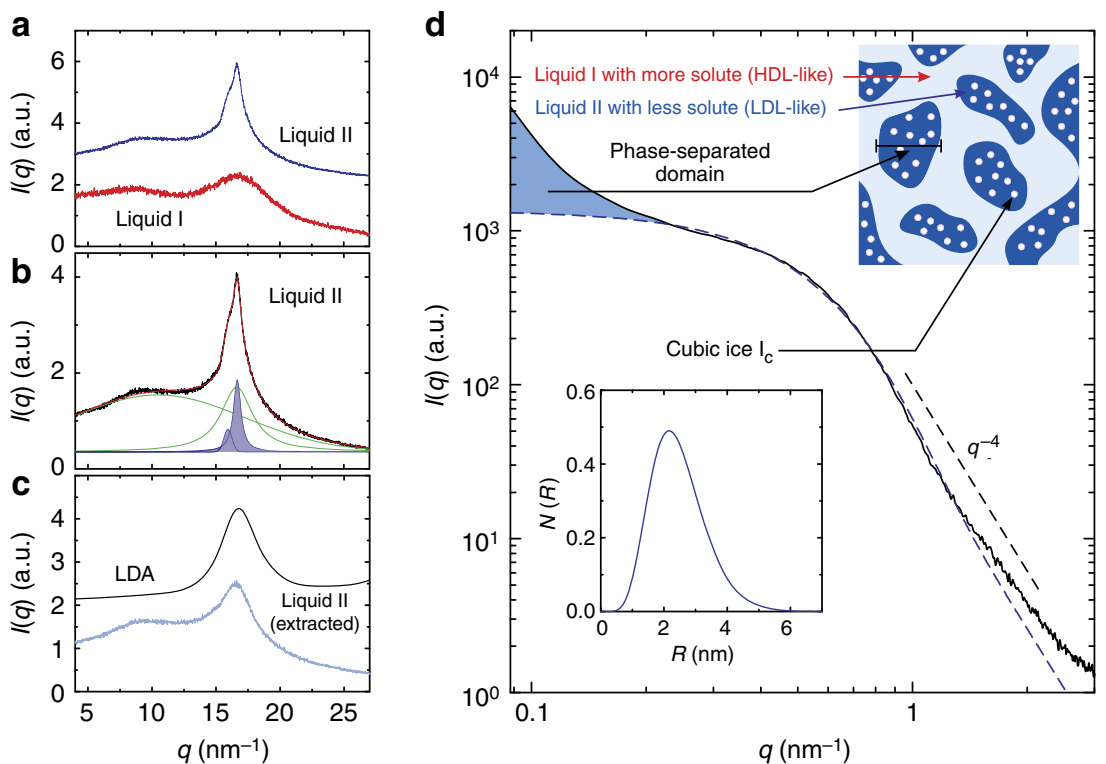

Figure 3 | Micro- and mesoscopic structures of liquid I and II. (a) X-ray scattering intensity I(q) of liquid I and II for a water/xylitol solution $(c=0.125)$, measured at $189.5 \mathrm{~K}$. (b) The decomposition of $I(q)$ to extract the contribution from ice $I_{c}$. The blue areas represent the peaks from ice $I_{c}$ whereas the green lines represent those from a residual liquid containing both liquid I and II. (c) Comparison of the spectrum between LDA of pure water $^{42}$ and liquid II obtained by removing the contribution from ice $I_{c}($ see $\mathbf{b})$. (d) $I(q)$ of liquid II coexisting with liquid I and cubic ices in a smallangle regime. The blue dashed line indicates the result of fitting of ice $I_{c}$. The black dashed line represents the Porod law $\left(I(q) \propto q^{-4}\right.$ for high $\left.q\right)$. Here we employed a polydisperse sphere model with the Schultz-Zimm size distribution for the fitting. The bottom inset shows the number distribution of the radius of the ice $I_{c}$ particles. The blue area shows the excess scattering from phase-separated domains. The upper inset is a schematic pattern formed by LLT-induced demixing.

Raman spectroscopy measurements. To elucidate a key factor controlling the solubility of a solute in liquid II, we investigated the effect of the solute type on the hydrogen-bonding state of water in liquid I and II (both with and without demixing) by using Raman spectroscopy (see Methods). Figure 4a,b shows polarized Raman spectra around the OD-stretching band of liquids I and II, respectively, for various heavy water solutions. Note that heavy water is used to separate the OD-stretching modes of water from the $\mathrm{OH}$-stretching modes of solute molecules. The spectra of liquid I and II (including the demixed cases) resemble those of HDA and LDA, respectively ${ }^{43}$, suggesting that liquid I is a low-S liquid whereas liquid II is a high-S liquid. We can see that the position of the Raman peak linked to tetrahedral ordering shifts towards a higher frequency and its intensity decreases with increasing the molecular weight of a solute. This clearly indicates that a solute with higher molecular weight leads to stronger distortion of the tetrahedral structure in water, that is, stronger frustration effects on tetrahedral ordering.

\section{Discussion}

First, we discuss the physical factor determining whether LLT accompanies demixing or not. In Fig. 5, we give a simple explanation for the origin of LLT-induced demixing. For solutes with lower molecular weight (ethylene glycol and 1,3-propanediol), tetrahedral order is much more developed due to the weak frustration effect of the solutes on it (see Fig. 4). We argue that too high tetrahedral order results in demixing upon LLT (see Supplementary Note 2) because the stabilization of tetrahedral order by expelling solute molecules lowers the free energy more efficiently than the mixing entropy gain does: demixing induced by a high degree of tetrahedral ordering. For solutes with higher molecular weight than 1,2,4-butanetriol, on the other hand, the increase of molecular weight leads to the increase of the dispersion force between solute molecules as well as the decrease of the mixing entropy gain. These factors together with frustration effects on tetrahedral ordering induce demixing: molecular size-induced demixing. LLT without demixing may be realized in a special condition where frustration effects on tetrahedral ordering and molecular size effects of a solute are both weak. We speculate that LLT with/without demixing can be generally controlled by the competition between these two physical factors.

So far we have discussed LLT of aqueous solutions for a few examples, focusing on the difference between LLT with and without demixing. Now we extract a universal feature of LLTs observed in all 14 solutes we investigated, which does not depend on whether LLT accompanies demixing or not. This insensitivity of the behaviour to demixing is a consequence of the causality that LLT takes place first and demixing is induced by it. Figure $6 a$ shows the $c$ - $T$ phase diagram for aqueous solutions of all the polyols and sugars we investigated. The depression of the melting point $T_{\mathrm{m}}$ and the spinodal temperature of LLT, $T_{\mathrm{SD}}$, with increasing $c$ means that the increase in the solute concentration lowers the free energy of liquid I because of solute-water interactions with an expense of the loss of tetrahedral order. It was previously shown $24,44,45$ that for various aqueous solutions the melting lines of ices and the homogeneous nucleation lines can be nicely collapsed on the two master curves, respectively, by using the water activity as the control parameter. Similarly, we experimentally found that the slope of the spinodal line of LLT is correlated to that of the melting line of ice for the solution, suggesting that the spinodal line is simply determined by the dilute limit of the spinodal temperature, $T_{\mathrm{SD}}(c=0)$, and the water-solute interaction parameter, $\chi_{\text {ws }}$. This motivates us to extend our two-order-parameter model of water ${ }^{9,36}$ to aqueous solutions for describing LLT in aqueous organic solutions. We also apply a similar extension to water/salt mixtures for 


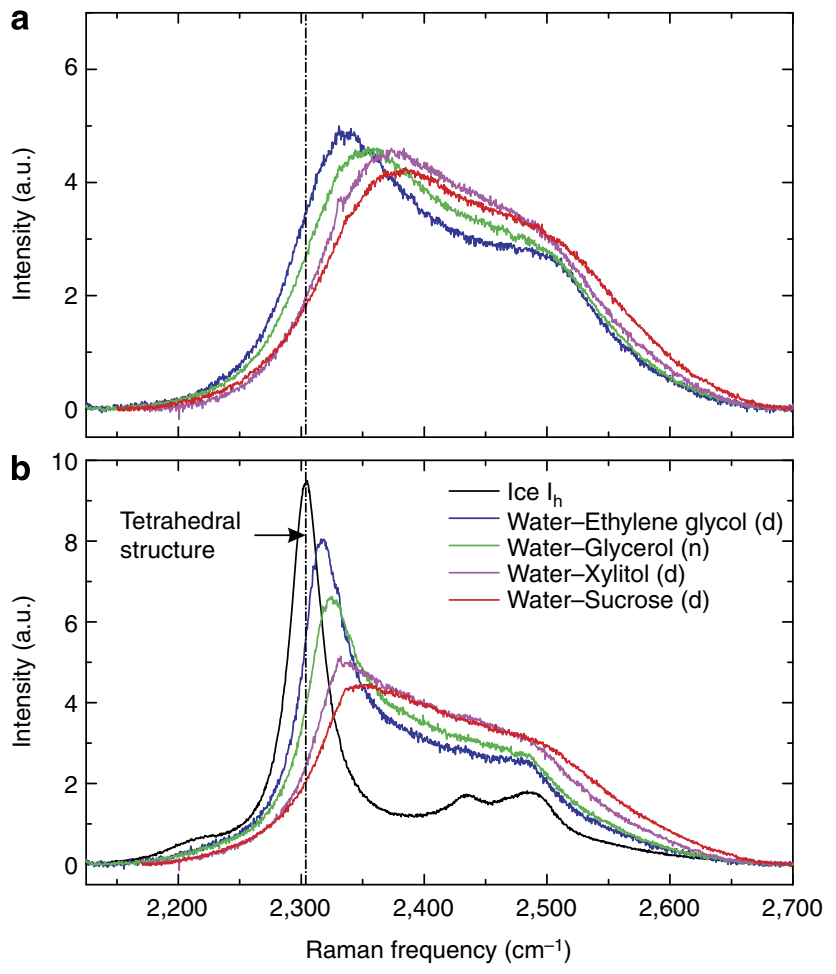

Figure 4 | Hydrogen-bonding states in liquid I and II. (a) Polarized Raman spectra around the OD-stretching bands of liquid I (low-S liquid): a water/ethylene glycol mixture at $162 \mathrm{~K}(c=0.215)$, a water/glycerol mixture at $173 \mathrm{~K}(c=0.185)$, a water/xylitol mixture at $189.5 \mathrm{~K}(c=0.135)$ and a water/sucrose mixture at $201.5 \mathrm{~K}(c=0.083)$. The colour of each curve is the same as that in panel $\mathbf{b}$. (b) The same spectra of liquid II (high-S liquid) with and without demixing. ( $n$ ) and (d) in the legend indicate LLT without and with demixing, respectively. Note that this applies only to (b). Here we also show the spectrum of pure ice $I_{h}$ as a reference. The vertical dash-dot lines in $\mathbf{a}$ and $\mathbf{b}$ represent the location of the strongest OD-stretching band of ice $\mathrm{I}_{\mathrm{h}}$ crystal.

describing the viscosity anomaly ${ }^{46,47}$. In relation to this, it is worth mentioning that we recently verified the validity of our two-state model for TIP4P/2005 water ${ }^{48}$. We successfully estimated the fraction of locally favoured structures of water, $S$, on a microscopic structural basis by using translational order in the second shell as the relevant structural order parameter, and showed that the two-order-parameter model can well describe thermodynamic anomalies of the water on a quantitative level.

According to our two-order-parameter model, the molar Gibbs free energy of an aqueous solution can be represented as

$$
\begin{aligned}
G(c, S)= & c G_{\mathrm{sol}}^{0}+(1-c)\left(S H_{S}+(1-S) H_{\rho}\right) \\
& +R T(c \ln c+(1-c) \ln (1-c))+R T(1-c) \\
& \times\left(S \ln \frac{S}{g_{S}}+(1-S) \ln \frac{1-S}{g_{\rho}}\right)+\chi_{\mathrm{ws}} c(1-c) \\
& +\chi_{\mathrm{LN}}(c)(1-c) S(1-S),
\end{aligned}
$$

where $R$ is the gas constant and the subscripts, $\rho$ and $S$, represent normal-liquid and locally favoured structures in water, respectively. The molar Gibbs free energy of the solute is defined as $G_{\mathrm{sol}}^{0}$. We set $H_{j}$ and $g_{j}$ as the enthalpy and degeneracy of each state $(j=\rho$ or $S)$. The enthalpy $H_{j}$ is given by $E_{j}+P v_{j}$, where $E_{j}$ and $v_{j}$ are the energy and specific volume of each state. We assume that $g_{S}<<g_{\rho}$ because locally favoured structures have rather unique structures, whereas normal-liquid structures have many possible

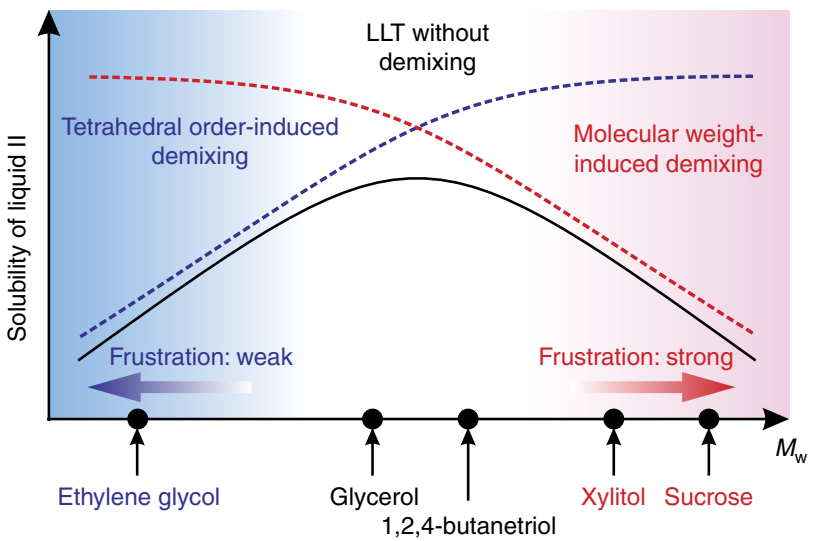

Figure 5 | A schematic for the origin of LLT-induced demixing. Very small molecular weight solutes only weakly perturb tetrahedral order of water. In such a case, the free energy gain by forming a higher $S$ state with less solute is larger than the mixing entropy gained by homogeneous mixing. On the other hand, larger molecular weight solutes strongly perturb tetrahedral order of water and furthermore they have stronger attractions between them and smaller mixing entropy. Thus, demixing takes place upon LLT because the solutes mix better to a low-S liquid rather than a high-S liquid. LLT without demixing only takes place for intermediate molecular weights for which there is a subtle balance between frustration and mixing entropy effects. This qualitative picture need to be confirmed on a more quantitative level in the future.

configurations ${ }^{9,36}$. Here $\chi_{w s}$ is the water-solute interaction parameter and $\chi_{\mathrm{LN}}$ is the interaction parameter between $\rho$ and $S$ structures.

The interaction parameter $\chi_{\mathrm{LN}}$, which governs LLT in our model, has a concentration dependence, as the probability that one water molecule can interact with nearest-neighbour water molecules, which is characterized by $\chi_{\text {ws }}$, decreases with an increase in $c$. For an ideal solution $(c<0.025$, dilute solutions in our system, see Fig. $6 \mathrm{a}), \chi_{\mathrm{LN}}(c)=(1-c) \chi_{\mathrm{LN}}^{0}$, where $\chi_{\mathrm{LN}}^{0}=\chi_{\mathrm{LN}}(0)$. Strictly speaking, however, we should use the relation $\chi_{\mathrm{LN}}(c)=\chi_{\mathrm{LN}}^{0} \exp \left(\Delta \mu_{\mathrm{aq}} / R T\right)=\chi_{\mathrm{LN}}^{0} a_{\mathrm{w}}$, which takes into account the fact that the attractive solute-water interactions increase the solute concentration around a water molecule than the simple average. Here $\Delta \mu_{\mathrm{aq}}=\mu(c)-\mu(0)$ is a difference in the chemical potential of water between the aqueous solution and pure water at the same temperature and pressure. In the above, the relation $a_{\mathrm{w}}=\exp \left(\Delta \mu_{\mathrm{aq}} / R T\right)$ is used.

We note that the chemical potential of water in an aqueous solution $\mu(c)$ is given by $G-c(\partial G / \partial c)$. Thus, the explicit forms of $G(c, S)$ and $\mu(c)$ (or $\Delta \mu_{\mathrm{aq}}$ ) are obtained by solving the differential equation with this expression of $\chi_{\mathrm{LN}}(c)$ in a self-consistent manner. We can analytically solve this differential equation by ignoring higher order terms, $c^{n}(n \geq 3)$, which is a reasonable assumption in our $c$ range, and obtain the following relation:

$$
\begin{aligned}
\chi_{\mathrm{LN}}(c) & =\chi_{\mathrm{LN}}^{0}(1-c) \exp \left(\frac{\left(\chi_{\mathrm{ws}}-\chi_{\mathrm{LN}}^{0} S(1-S)\right) c^{2}}{R T}\left(\frac{1}{1+\chi_{\mathrm{LN}}^{0} S(1-S) / R T}\right)\right) \\
& =\chi_{\mathrm{LN}}^{0}(1-c) \exp \left(\left(\frac{\chi_{\mathrm{ws}}}{R T}-\frac{T_{\mathrm{SD}}^{0}}{2 T}\right) c^{2}\left(1+\frac{T_{\mathrm{SD}}^{0}}{2 T}\right)^{-1}\right) .
\end{aligned}
$$

in the above, we use the fact that $T_{\mathrm{SD}}$ of pure water $(c=0)$ is given by $T_{\mathrm{SD}}^{0}=2 \chi_{\mathrm{LN}}^{0} S(1-S) / R$. This means that the water activity $a_{\mathrm{w}}$ is given by

$$
a_{\mathrm{w}}=(1-c) \exp \left(\left(\frac{\chi_{\mathrm{ws}}}{R T}-\frac{T_{\mathrm{SD}}^{0}}{2 T}\right) c^{2}\left(1+\frac{T_{\mathrm{SD}}^{0}}{2 T}\right)^{-1}\right) .
$$

now we show that both $T_{\mathrm{m}}(c)$ and $T_{\mathrm{SD}}(c)$ can be scaled by the water activity $a_{\mathrm{w}}=\exp \left(\Delta \mu_{\mathrm{aq}} / R T\right)$. As the difference $\Delta \mu_{\mathrm{aq}}$ is 
a

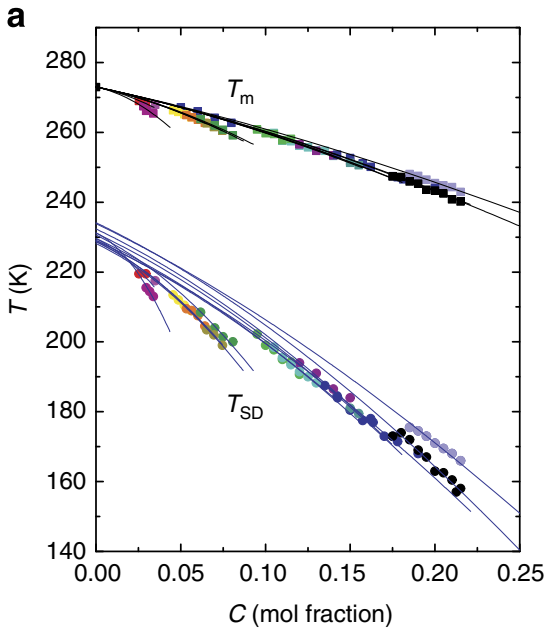

b

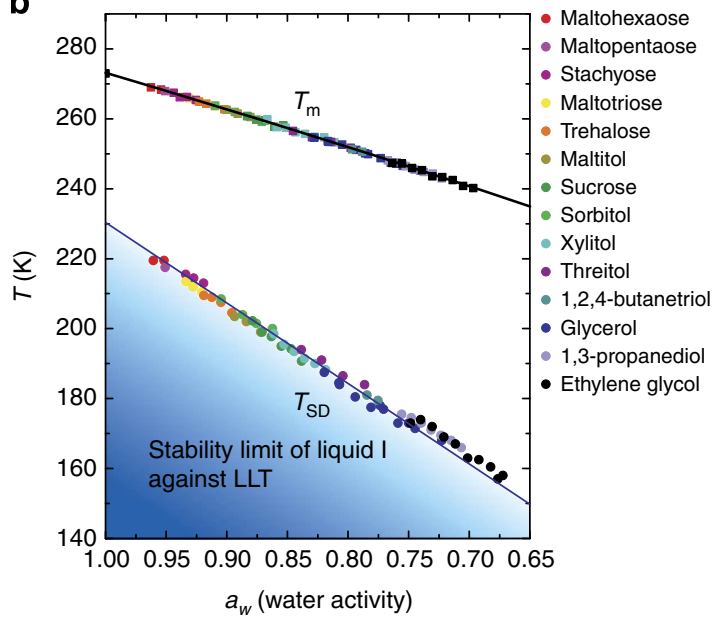

Figure 6 | Phase diagram of aqueous solutions. (a) Solute concentration versus temperature $(c-T)$ phase diagram of aqueous solutions. $T_{m}$, ice melting temperature (filled squares); $T_{\mathrm{SD}}$, LLT spinodal temperature of aqueous solutions (filled circles). The black and blue solid lines show the melting and spinodal line fitted by the combination of equations (3) and (5) (melting), and that of equations (3) and (6) (LLT spinodal), respectively. The errors of each point is $\pm 2 \mathrm{~K}$ for $T_{\mathrm{SD}} \pm 0.5 \mathrm{~K}$ for $T_{\mathrm{m}}$ and $\pm 0.0005 \mathrm{in}$ c. The values of the fitting parameters are listed in Supplementary Table S1. (b) Water activity versus temperature $\left(a_{w}-T\right)$ phase diagram of aqueous solutions. The blue solid straight line indicates the spinodal line scaled by equation ( 6 ). The black solid nearly straight line indicates the melting line obtained by equation (5). The blue area indicates the unstable region of liquid I against liquid II.

identical to that between ice and water, $\Delta \mu_{\mathrm{iw}}$, on the melting (equilibrium) point, the melting point depression is also determined by $\Delta \mu_{\mathrm{aq}}$ as follows:

$$
\Delta \mu_{\mathrm{aq}}=\Delta \mu_{\mathrm{iw}}=\Delta H_{\mathrm{f}}-T_{\mathrm{m}} \Delta S_{\mathrm{f}}=\frac{\Delta H_{\mathrm{f}}}{T_{\mathrm{m}}^{0}}\left(T_{\mathrm{m}}^{0}-T_{\mathrm{m}}\right),
$$

where $\Delta H_{\mathrm{f}}=6.01 \mathrm{~kJ} \mathrm{~mol}^{-1}$ is the enthalpy of fusion of pure ice, $\Delta S_{\mathrm{f}}$ is the entropy of fusion of pure ice, $T_{\mathrm{m}}^{0}$ is the melting temperature of pure ice $(273 \mathrm{~K})$ and $T_{\mathrm{m}}$ is that of ices in an aqueous solution. Here we used a relation of $\Delta S_{\mathrm{f}}=\Delta H_{\mathrm{m}} / T_{\mathrm{m}}^{0}$. Thus, we obtain the following relation ${ }^{23,49}$ :

$$
\frac{1}{T_{\mathrm{m}}}=\frac{1}{T_{\mathrm{m}}^{0}}-\frac{R}{\Delta H_{\mathrm{f}}} \ln a_{\mathrm{w}} .
$$

On the other hand, the spinodal temperature of LLT, $T_{\mathrm{SD}}$, is given from the condition of $\partial^{2} G / \partial S^{2}=0$ as

$$
T_{\mathrm{SD}}(c)=\frac{2 \chi_{\mathrm{LN}}(c)}{R} S(1-S)=T_{\mathrm{SD}}^{0} a_{\mathrm{w}}
$$

Equations (5) and (6) imply that $T_{\mathrm{m}}(c)$ and $T_{\mathrm{SD}}(c)$ should be the functions of only $a_{\mathrm{w}}$. Thus, we fit these relations (5) and (6) with $a_{\mathrm{w}}$ given by equation (3) to the data of the $c$ dependence of $T_{\mathrm{m}}(c)$ and $T_{\mathrm{SD}}(c)$, respectively. From the fittings shown in Fig. 6a, we determine $T_{\mathrm{SD}}^{0}$ and $\chi_{\mathrm{ws}}$, which are the only adjustable parameters (see Methods and Supplementary Table S1). Here we note that equation (5) is the same as that in previous studies ${ }^{23,49}$ and the melting point depression is consistent with that reported there.

Figure 6b shows the phase diagram scaled by $a_{\mathrm{w}}$ instead of $c$. Consistent with the above predictions, we find that $T_{\mathrm{SD}}$ of all the aqueous solutions studied can be collapsed on a single straight line in this plot and well fitted by equation (6). Curiously, $T_{\mathrm{SD}}\left(a_{\mathrm{w}}=1\right)$, or $T_{\mathrm{SD}}^{0}$, estimated is located $\sim 230 \mathrm{~K}$, which is very close to the critical temperature of pure water $(228 \mathrm{~K})$, which has often been used for power law fittings of various thermodynamic variables $^{2}$. The above finding suggests the key role of water in LLT in the aqueous solutions. The water activity given by equation (3) can be interpreted as the effective concentration of water molecules that are free from hydrations to solute molecules or the distortion of hydrogen bonding and capable of tetrahedral ordering. The fact that $T_{\mathrm{SD}}$ can be scaled by $a_{\mathrm{w}}$ independent of the types of solute molecules is suggestive of the presence of LLT even in pure water, but this point needs further careful study to be confirmed. We note that the solute molecules stabilize liquid II by suppressing the formation of ice $\mathrm{I}_{\mathrm{c}}$ and ice $\mathrm{I}_{\mathrm{h}}$ and also increase the viscosity high enough for the kinetics of LLT to be followed.

The above results indicate that LLTs of the aqueous organic solutions are universally controlled by the water activity $a_{\mathrm{w}}$ alone. The pressure dependence of the spinodal, $T_{\mathrm{SD}}(P)$, is also expected to be scaled by $a_{\mathrm{w}}$ (ref. 23). However, whether the change in $a_{\mathrm{w}}$ can be 'directly' translated to that in $P$ or not is a delicate issue because the effects of $P$ on water structures are global (or homogeneous), whereas those of solutes are local. Further study is highly desirable for answering this interesting question.

\section{Methods}

Samples. In this study, we used de-ionized water and heavy water (deuterium oxide) purchased from ACROS ORGANICS (D-enrichment > 99.95\%). Solutes used are as follows: ethylene glycol (Wako Pure Chemical Industries, Ltd.), glycerol (Merck Co., Inc), 1,2,4-butanetriol and trehalose dihydrate (Tokyo Chemical Industry Co., ltd), maltohexaose (ACROS ORGANICS), 1,3-propanediol and sucrose (Kanto Chemical Co., Inc.), xylitol, maltitol and maltopentaose (SIGMA), and threitol, sorbitol, stachyose hydrate and maltotriose hydrate (ALDRICH).

Optical microscopy observation. We observed the transformation process between liquid I and II with phase contrast microscopy (Olympus, BH2-UMA). A sample was sandwiched between two cover glasses, whose gap was controlled to be $\sim 10 \mu \mathrm{m}$. The temperature was controlled within $\pm 0.1 \mathrm{~K}$ by a computer-controlled hot stage (Linkam LK-600PH) equipped with a cooling unit (Linkam L-600A).

Thermal measurements. We measured the heat flux during the transformation with a differential scanning calorimeter (Mettler Toledo, DSC-822e), which is capable of complex (ac) heat capacity measurements. The ac measurements were performed with a modulated heating rate, whose average, period and amplitude are $1 \mathrm{~K} \mathrm{~min}^{-1}$, $60 \mathrm{~s}$ and $0.16 \mathrm{~K}$, respectively.

X-ray scattering measurements. Small-angle (SAXS) and wide-angle X-ray scattering (WAXS) measurements were carried out by using a SAXSess camera (Anton Parr), covering the $q$ range from $0.088 \mathrm{~nm}^{-1}$ to $27 \mathrm{~nm}^{-1}$.

Raman spectroscopy measurements. Raman spectroscopy measurements were made by using a micro Raman diffraction meter (Jobin Yvon Inc., T64000). We used the beam size of $\sim 1 \mu \mathrm{m} \times 1 \mu \mathrm{m}$ to have a high spatial resolution. 
Analysis of SAXS spectra of cubic ice $\mathbf{I}_{\mathbf{c}}$. We analyse $I(q)$ of cubic ice $\mathrm{I}_{\mathrm{c}}$ in a low $q$ regime by regarding ice crystals as polydisperse hard spheres whose radii $r$ have the Schultz-Zimm distribution $N(r)$ :

$$
\begin{gathered}
I(q)=4 \pi \int_{0}^{\infty} I_{\text {sphere }}(r, q) N(r) d r, \\
N(r)=\frac{1}{r_{\mathrm{c}}}\left(\frac{1}{r_{\mathrm{c}}}\right)^{k-1} \frac{k^{k} \exp \left(-k r / r_{\mathrm{c}}\right)}{\Gamma(k)},
\end{gathered}
$$

where $I_{\text {sphere }}(r, q)$ is the form factor of a hard sphere, $r_{\mathrm{c}}$ defines $r$ at which the size distribution has a peak for large values of $k=1 / \sigma^{2}$ ( $\sigma$ being the variance) and $\Gamma$ is the Gamma function.

Analysis of the $\boldsymbol{c}$ dependence of $\boldsymbol{T}_{\mathbf{m}}$ and $\boldsymbol{T}_{\mathbf{S D}}$. We fitted the data of $T_{\mathrm{m}}(c)$ and $T_{\mathrm{SD}}(c)$ shown in Fig. 6a, using equations (5) and (6) with $a_{\mathrm{w}}$ given by equation (3) under a constraint that $T_{\mathrm{SD}}^{0}$ and $\chi_{\mathrm{ws}}$ are to be common to all the sets of data. Supplementary Table S1 summarizes the results of the fittings for all aqueous solutions we investigated. Then, we obtain $T_{\mathrm{SD}}^{0}=230.4 \mathrm{~K}$ by fitting equation (6) to the data after scaling by $a_{\mathrm{w}}$ (Fig. 6b).

\section{References}

1. Debenedetti, P. G. Metastable Liquids (Princeton University Press, 1997).

2. Debenedetti, P. G. Supercooled and glassy water. J. Phys. Condens. Matter 15, R1669-R1726 (2003).

3. Mishima, O. \& Stanley, H. E. The relationship between liquid, supercooled and glassy water. Nature 396, 329-325 (1998).

4. Angell, C. A. Insights into phases of liquid water from study of its unusual glass-forming properties. Science 319, 582-587 (2008).

5. Tanaka, H. Simple view of waterlike anomalies of atomic liquids with directional bonding. Phys. Rev. B 66, 064202 (2002)

6. Soper, A. K. Recent water myths. Pure Appl. Chem. 82, 1855-1867 (2010).

7. Nilsson, A., Huang, C. \& Pettersson, L. G. M. Fluctuations in ambient water. J. Mol. Liq. 176, 2-16 (2012).

8. Clark, G. N. I., Cappa, C. D., Smith, J. D., Saykally, R. J. \& Head-Gordon, T. The structure of ambient water. Mol. Phys. 108, 1415-1433 (2010).

9. Tanaka, H. Bond orientational order in liquids: Towards a unified description of water-like anomalies, liquid-liquid transition, glass transition, and crystallization. Eur. Phys. J. E 35, 113 (2012).

10. Mishima, O., Calvert, L. D. \& Whalley, E. An apparent first-order transition between two amorphous phases of ice induced by pressure. Nature 314, 76-78 (1985).

11. Tanaka, H. Thermodynamic anomaly and polyamorphism of water. Europhys. Lett. 50, 340-346 (2000)

12. Poole, P. H., Sciortino, F., Essmann, U. \& Stanley, H. E. Phase behaviour of metastable water. Nature 360, 324-328 (1992).

13. Limmer, D. T. \& Chandler, D. The putative liquid-liquid transition is a liquid-solid transition in atomistic models of water. J. Chem. Phys. 135, 134503-134512 (2011).

14. Limmer, D. T. \& Chandler, D. The putative liquid-liquid transition is a liquid-solid transition in atomistic models of water. ii. J. Chem. Phys. 138, 214504 (2013).

15. Liu, Y., Panagiotopoulos, A. Z. \& Debenedetti, P. G. Low-temperature fluidphase behavior of ST2 water. J. Chem. Phys. 131, 104508 (2009).

16. Liu, Y., Palmer, J. C., Panagiotopoulos, A. Z. \& Debenedetti, P. G. Liquid-liquid transition in ST2 water. J. Chem. Phys. 137, 214505-214514 (2012).

17. Poole, P. H., Bowles, R. K., Saika-Voivod, I. \& Sciortino, F. Free energy surface of ST2 water near the liquid-liquid phase transition. J. Chem. Phys. 138, 034505-034511 (2013).

18. Kesselring, T. A. et al. Finite-size scaling investigation of the liquid-liquid critical point in ST2 water and its stability with respect to crystallization. J. Chem. Phys. 138, 224506-224518 (2013).

19. Mallamace, F. et al. Transport properties of supercooled confined water. Eur. Phys. J. Special Topics 161, 19-33 (2008).

20. Giovambattista, N., Rossky, P. J. \& Debenedetti, P. G. Effect of pressure on the phase behavior and structure of water confined between nanoscale hydrophobic and hydrophilic plates. Phys. Rev. E 73, 041604-041617 (2006).

21. Giovambattista, N., Rossky, P. J. \& Debenedetti, P. G. Effect of temperature on the structure and phase behavior of water confined by hydrophobic, hydrophilic, and heterogeneous surfaces. J. Phys. Chem. B 113, 13723-13734 (2009).

22. Murata, K. \& Tanaka, H. Liquid-liquid transition without macroscopic phase separation in a water-glycerol mixture. Nat. Mater. 11, 436-443 (2012).

23. Koop, T., Luo, B., Tsias, A. \& Peter, T. Water activity as the determinant for homogeneous ice nucleation in aqueous solutions. Nature 406, 611-614 (2000).

24. Koop, T. Homogeneous ice nucleation in water and aqueous solutions. Z. Phys. Chem. 218, 1231-1258 (2004).

25. Kurita, R., Murata, K. \& Tanaka, H. Control of fluidity and miscibility of a binary liquid mixture by the liquid-liquid transition. Nat. Mater. 7, 647-652 (2008).

26. Angell, C. A. \& Sare, E. J. Liquid-liquid immiscibility in common aqueous salt solutions at low temperatures. J. Chem. Phys. 49, 4713-4714 (1968).
27. Chatterjee, S. \& Debenedetti, P. G. Fluid-phase behavior of binary mixtures in which one component can have two critical point. J. Chem. Phys. 124, 154503-154512 (2006).

28. Suzuki, Y. \& Mishima, O. Sudden switchover between the polyamorphic phase separation and the glass-to-liquid transition in glassy $\mathrm{LiCl}$ aqueous solutions. $J$. Chem. Phys 138, 084507-084515 (2013).

29. Le, L. \& Molinero, V. Nanophase segregation in supercooled aqueous solutions and their glasses driven by the polyamorphism of water. J. Phys. Chem. A 115, 5900-5907 (2011).

30. Corradini, D., Strekalova, E. G., Stanley, H. E. \& Gallo, P. Microscopic mechanism of protein cryopreservation in an aqueous solution with trehalose. Sci. Rep. 3, 1218 (2013).

31. Murray, B. J., Knopf, D. A. \& Bertram, A. K. The formation of cubic ice under conditions relevant to earth's atmosphere. Nature 434, 202-205 (2005).

32. Jenniskens, P. \& Blake, D. F. Structural transitions in amorphous water ice and astrophysical implications. Science 265, 753-756 (1994)

33. Tanaka, H., Kurita, R. \& Mataki, H. Liquid-liquid transition in the molecular liquid triphenyl phosphite. Phys. Rev. Lett. 92, 025701-025704 (2004).

34. Kurita, R. \& Tanaka, H. On the abundance and general nature of the liquidliquid phase transition in molecular systems. J. Phys. Condens. Matter 17, L293-L302 (2005).

35. Tanaka, H. Two-order-parameter description of liquids: Critical phenomena and phase separation of supercooled liquids. J. Phys. Condens. Matter 11, L159-L168 (1999).

36. Tanaka, H. General view of a liquid-liquid phase transition. Phys. Rev. E 62, 6968-6976 (2000).

37. Hayashi, Y., Puzenko, A. \& Feldman, Y. Ice nanocrystals in glycerol-water mixtures. J. Phys. Chem. B 109, 16979-16981 (2005).

38. Limmer, D. T. \& Chandler, D. Corresponding states for mesostructure and dynamics of supercooled water. Preprint at http://arxiv.org/abs/1305.1382 (2013).

39. Langer, J. S. Instabilities and pattern formation in crystal growth. Rev. Mod. Phys. 52, 1-28 (1980).

40. Hallbrucker, A. \& Mayer, E. Calorimetric study of the vitrified liquid water to cubic ice phase transition. J. Phys. Chem. 91, 503-505 (1987).

41. Onuki, A. Phase Transition Dynamics (Cambridge University Press, 2002).

42. Schober, H. et al. Crystal-like high frequency phonons in the amorphous phases of solid water. Phys. Rev. Lett. 85, 4100-4103 (2000).

43. Mishima, O. \& Suzuki, Y. Propagation of the polyamorphic transition of ice and the liquid-liquid critical point. Nature 419, 599-603 (2002).

44. Rasmussen, D. H. Ice formation in aqueous systems. J. Microsc. 128, 167-174 (1982).

45. Rasmussen, D. H. \& Liang, M. T. A theory of nucleation of ice. In Symposium on Nucleation and Crystallization in Glasses and Liquids. pp. 85-91 (American Ceramic Society, 1993).

46. Kobayashi, M. \& Tanaka, H. Possible link of the V-shaped phase diagram to the glass-forming ability and fragility in a water-salt mixture. Phys. Rev. Lett. 106, 125703 (2011).

47. Kobayashi, M. \& Tanaka, H. Relationship between the phase diagram, the glassforming ability, and the fragility of a water/salt mixture. J. Phys. Chem. B 115, 14077-14090 (2011).

48. Russo, J. \& Tanaka, H. Understanding water's anomalies with locally favored structures. Preprint at http://arxiv.org/abs/1308.4231 (2013).

49. Koop, T. The water activity of aqueous solutions in equilibrium with ice. Bull. Chem. Soc. Jpn 75, 2587-2588 (2002).

\section{Acknowledgements}

We thank John Russo for a critical reading of our manuscript. We are also grateful to David Chandler and David Limmer and to Yuri Feldman, Anna Greenbaum, Alexander Puzenko and Alexei Sokolov for stimulating discussions on the nature of the transition This study was partly supported by Grants-in-Aid for Scientific Research (S) and Specially Promoted Research from JSPS and by Aihara Project, the FIRST programme from JSPS, initiated by CSTP.

\section{Author contributions}

H.T. conceived and supervised the project, K.M. performed experiments, K.M. and H.T. discussed and wrote the manuscript.

\section{Additional information}

Supplementary Information accompanies this paper at http://www.nature.com/ naturecommunications

Competing financial interests: The authors declare no competing financial interests

Reprints and permission information is available online at http://npg.nature.com/ reprintsandpermissions/

How to cite this article: Murata, K. and Tanaka, H. General nature of liquid-liquid transition in aqueous organic solutions. Nat. Commun. 4:2844 doi: 10.1038/ncomms3844 (2013). 